\title{
Effectiveness of headgear in a pilot study of under 15 rugby union football
}

School of Safety Science, University of New South Wales, Sydney, Australia 2052 A S McIntosh

Centre for Sports Medicine Research and Brain Research Institute, University of Melbourne, Parkville, Victoria, Australia 3052

P McCrory

Correspondence to: Dr McIntosh

a.mcintosh@unsw.edu.au

Accepted 19 February 2001

\author{
A S McIntosh, P McCrory
}

\begin{abstract}
Objective-To determine whether protective headgear reduced the incidence of concussion in a pilot study of under 15 rugby union.

Methods-Sixteen under 15 rugby union teams were recruited from three interschool competitions in metropolitan Sydney and the adjacent country region. A prospective study was undertaken over a single competitive season. The study had two arms: a headgear arm and a control arm. Headgear wearing rates and injury data were reported to the investigators and verified using spot checks.

Results-A total of 294 players participated in the study. There were 1179 player exposures with headgear and 357 without headgear. In the study time frame, there were nine incidences of concussion; seven of the players involved wore headgear and two did not. There was no significant difference between concussion rates between the two study arms.

Conclusions-Although there is some controversy about the desirability of wearing protective headgear in football, this pilot study strongly suggests that current headgear does not provide significant protection against concussion in rugby union at a junior level.

(Br f Sports Med 2001;35:167-169)
\end{abstract}

Keywords: rugby union; headgear; helmets; concussion; adolescents

The use of helmets or head protectors to reduce traumatic brain injury in sport is a controversial area. Whereas in some sports-for example, cycling, ice hockey-there is published evidence for a protective effect, ${ }^{1-6}$ in others-for example, American football-the evidence is inconsistent. ${ }^{7-9}$ In part, this relates not to the impact characteristics of the helmets but rather the effect of other interventions, such as rule changes, which cloud the issue of the protective benefit.

In rugby union, specific rules limit the nature and type of helmets that may be worn in matches. ${ }^{10}$ Although there are contrasting opinions about the desired or intended function of headgear-for example, protection against abrasion/laceration versus protection against brain injury-it is unclear whether the currently permitted rugby headgear performs either function. ${ }^{11}{ }^{12} \mathrm{~A}$ recent laboratory study of the impact energy attenuation properties of headgear showed that current models have a very limited capacity to reduce the likelihood of concussion. ${ }^{12}$

As laboratory tests cannot precisely replicate the conditions in real head impacts, this study was designed to trial headgear in the field and, in particular, to determine whether the laboratory tests had underestimated the protective capacity of headgear.

The incidence of head injury and concussion in rugby has been extensively studied in many countries. ${ }^{13-18}$ The incidence of concussion in prospective studies is about 3.4 per 1000 player hours exposure and concussion accounts for about $5 \%$ of the total injuries. ${ }^{14}$ Given the concerns of both acute and long term sequelae from concussive injury, any injury prevention strategies that will decrease this injury burden deserve critical analysis. Helmets have been suggested anecdotally as one means by which injuries may be reduced. This paper examines this premise in a controlled trial.

\section{Methods}

This is a prospective pilot study of the protective effectiveness of football headgear conducted in an official interschool under 15 rugby competition. There were two study arms, a headgear arm and a control arm.

All teams from interschool under 15 rugby competitions, encompassing 22 schools, in the Sydney Metropolitan Area and adjacent Southern Highlands region were invited to participate in the study. A total of 16 schools volunteered to participate. At each school, the under $15 \mathrm{~A}$ grade team was the team studied. We believe that the teams who chose to participate were representative of the sample as a whole and represent the elite schoolboy level of competition. Using a random number approach, nine teams were selected to wear headgear, and seven formed the non-helmeted control teams.

After agreement had been obtained from each school and team coach, players in each team were invited to participate in the study. Each player and their parents or guardians completed an informed consent form. The ethics and research committee of the University of New South Wales approved the study.

Two models of commercial rugby union headgear were provided to players not already wearing headgear in the headgear teams. As the study aim was to examine the effectiveness of headgear, not compare specific models, players were allowed to continue wearing the model of their choice. The two models, the Albion "BodyPro" and Madison "Elite Rugby", had been shown to perform in a similar fashion in laboratory tests of impact energy attenuation. ${ }^{12}$

Each team nominated a "recording officer" who documented details of player participation, 
headgear use, and head injury occurrence for each game. A simple injury information sheet was provided and was to be returned by fax to the investigators once a head injury had occurred. The recording officer was contacted regularly to obtain a status report and to confirm that no injuries had occurred. Team medical officers were contacted to verify injury diagnosis and the authors reviewed all injury reports. For the purpose of the study, an injury was defined as a traumatic event that resulted in the player missing game playing or training time.

The investigators attended three games a week to determine independently headgear wearing rates and maintain contact with the teams. All participating teams were reviewed at least once during the sampling period.

Six games were videotaped using a hand held digital camera to provide some control data for rate and location of head impacts between the two study arms. An unlikely, but confounding, possibility was that one group would receive a greater number of more severe head impacts, thereby increasing the likelihood of concussion independently of the intervention. The video was reviewed at the end of the season.

Rates of concussion were calculated for each group on the basis of the number of cases of concussion and the exposure-that is, total number of players participating in all games for each arm. A comparison of two proportions was undertaken for the unpaired case to obtain a standardised normal deviate. This was used to test the null hypothesis that there was no difference in rates of concussion between the two study arms.

\section{Results}

All 16 invited teams completed the study. A total of 294 players participated in a total of 1179 player exposures with headgear and 357 without headgear.

There was a good correlation $(r=0.95)$ between the headgear wearing rates reported by the team recording officer and those observed by the investigators for the 15 games attended. The investigators did not attend the entire game, so were not able to provide data on player replacements.

There were nine incidences of medically verified concussion; seven of the players were wearing headgear and two were not. One participant required brief observation in a hospital emergency department. No player suffered a catastrophic brain injury.

There was no significant difference $(p=0.48)$ between the crude injury rates of players with and without headgear. The standardised normal deviate, $\mathrm{z}$, was 0.0648 , with $95 \%$ confidence limits of 0.0092 and -0.0086 . Therefore the null hypothesis, that current headgear does not reduce the likelihood of concussion, was retained.

Six games were videotaped and examined to determine the nature of the head impacts. A total of 22 non-concussive head impacts could be observed on the video, 15 involving players with headgear. Of the 22 impacts, 14 were to the lateral head, two were frontolateral, and four were frontal. The remaining impacts were to the face, the occiput, and the top of the head. The impacting objects struck were the ground (five occasions), forearm/elbow (five occasions), chest (three occasions), and upper arm/ shoulder (three occasions), with the remainder by the leg and pelvis. The video analysis did not indicate that there was a difference in the type of head impact experienced by players in each study arm. Previously published research has documented the pathomechanics of concussive injury in this sport and the limitations of videoanalysis as an investigative tool. ${ }^{13}$

\section{Discussion}

While there is some controversy about the desirability of wearing protective headgear in football, this pilot study suggests that currently available commercial headgear does not provide protection against concussion in rugby union at a junior level.

The study is limited by the relatively small number of cases of concussion observed in the two arms during the study time. A longer study is in progress to increase the power of such observations. Nevertheless the study's findings reflect previous observations from a laboratory test-that is, the poor attenuation of impact energy observed for the range of protective headgear. $^{12}$

There are relatively few methods by which concussive brain injury may be minimised in sport. Unlike the musculoskeletal system, the brain cannot be conditioned to withstand injury. Thus extrinsic mechanisms of injury prevention are usually sought.

Protective headgear has been proposed to protect the head and theoretically reduce the risk of brain injury. In sports in which there is potential for high speed collisions or missile injuries - for example, baseball - or for falls on to hard surfaces-for example, gridiron, ice hockey-there is published evidence for the effectiveness of sport specific helmets in reducing head injuries. ${ }^{2419}$ For sports such as rugby, soccer, and Australian football, no sport specific helmets have been shown to be of benefit in reducing rates of head injury. In fact, most commercially available soft helmets fail to meet impact testing criteria that would be typical of sport related concussion. ${ }^{12}$

An interesting finding in the study was that total head impacts did not differ in the type or frequency between players in each study arm. Although the numbers were small (22 impacts studied by videoanalysis), it does suggest that the widely held belief that helmeted players are "targeted" or receive more hits to the head than normal from their opponents may be incorrect.

Arguably, the most important aspect of preventive care is the education of team doctors and others involved in athletic care with regard to on field recognition of concussive injury and the application of appropriate guidelines in returning athletes to sport safely. In the absence of an effective head protector or helmet suitable for rugby, this remains the current best practice.

We gratefully acknowledge the financial support of the New South Wales Sporting Injuries Committee and the Royal 


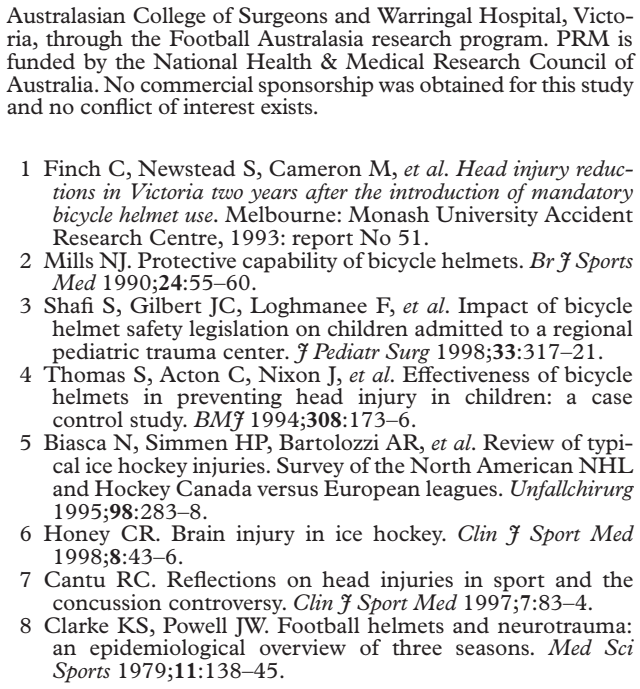
ria, through the Football Australasia research program. PRM is Australia. No commercial sponsorship was obtained for this study and no conflict of interest exists.

1 Finch C, Newstead S, Cameron M, et al. Head injury reductions in Victoria two years after the introduction of mandatory bicycle helmet use. Melbourne: Monash University Accident Research Centre, 1993: report No 51.

2 Mills NJ. Protective capability of bicycle helmets. Br F Sports Med 1990;24:55-60.

3 Shafi S, Gilbert JC, Loghmanee F, et al. Impact of bicycle helmet safety legislation on children admitted to a regional pediatric trauma center. F Pediatr Surg 1998;33:317-21.

4 Thomas S, Acton C, Nixon J, et al. Effectiveness of bicycle helmets in preventing head injury in children: a case control study. $B M \mathcal{F}$ 1994;308:173-6.

5 Biasca N, Simmen HP, Bartolozzi AR, et al. Review of typical ice hockey injuries. Survey of the North American NHL and Hockey Canada versus European leagues. Unfallchirurg 1995;98:283-8.

6 Honey CR. Brain injury in ice hockey. Clin $\mathcal{f}$ Sport Med

7 Cantu RC. Reflections on head injuries in sport and the concussion controversy. Clin $\mathcal{F}$ Sport Med 1997;7:83-4.

8 Clarke KS, Powell JW. Football helmets and neurotrauma: an epidemiological overview of three seasons. Med Sci Sports 1979;11:138-45

9 Virgin H. Cineradiographic study of football helmets and the cervical spine. Am $\mathcal{F}$ Sports Med 1980;8:310-17.

10 IRB. Laws of the game of rugby football. Dublin: International Rugby Board, 1996.

11 Wilson B. Protective headgear in rugby union. Sports Med 1998;25:333-7.

12 McIntosh A, McCrory P. Impact energy attenuation performance of football headgear. $\mathrm{Br} \mathcal{F}$ Sports Med 2000;34:337-42.

13 McIntosh A, McCrory P. The dynamics of concussive head impacts in rugby and Australian rules football. Med Sci Sports Exerc 2000;32:1980-5.

14 McCrory P. Neurological injuries in rugby and Australian rules football. In: Jordan B, Tsaris P, Warren R, eds. Sports neurology. 2nd ed. Philadelphia: Lippincott-Raven Publishers, 1998:441-9.

15 Gissane C, Jennings D, Cumine A, et al. Differences in the incidence of injury between rugby league forwards and backs. Aust f Sci Med Sport 1997;29:91-4.

16 Wekesa M, Asembo JM, Njororai WW. Injury surveillance in a rugby tournament. Br f Sports Med 1996;30:61-3.

17 Garraway M, Macleod D. Epidemiology of rugby football injuries. Lancet 1995;345:1485-7.

18 Edgar M. Tackling rugby injuries. Lancet 1995;345:1452-

19 Fekete JF. Severe brain injury and death following minor hockey accidents: the effectiveness of the safety helmets of amateur hockey players. Can Med Assoc F 1968;99:1234-9.

\section{British Association of Sport and Exercise Medicine in association with the National Sports Medicine Institute}

\section{Education programme 2001}

Injury management and medicine: thorax, groin, pelvis and hip

8-13 July

Lilleshall Hall National Sports Centre

General sports medicine

23-28 September

Lilleshall Hall National Sports Centre

Practical sport and medicine meeting

4-11 October

Club La Santa, Lanzarote

Families and non-delegates welcome; booking deadline 31 July

Diploma preparation course

October

Exact date and location to be confirmed

\section{BASEM Congress}

25-28 October

Vale of Glamorgan, Wales. Further details:

Tel/Fax: +44 (0)1928 732961; email: basemoffice@compuserve.com

Injury Management and Medicine: lower limb

18-23 November

Lilleshall Hall National Sports Centre

\section{Current Concepts}

2 December

Topic, date, and location to be confirmed

For further details of these courses please contact Mr Barry Hill, The National Sports Medicine Institute, c/o Medical College of St Bartholomew's Hospital, Charterhouse Square, London EC1M 6BQ.

Tel: 02072510583 (ext 237); Fax: 0207251 0774; email: barry.hill@nsmi.org.uk Web site: www.nsmi.org.uk 